\title{
Adaptación cultural de instrumentos de medida sobre estigma y enfermedad mental en la Ciudad de México
}

\author{
Jazmín Mora-Rios, ${ }^{1}$ Natalia Bautista-Aguilar, ${ }^{1}$ Guillermina Natera, ${ }^{1}$ Duncan Pedersen²
}

Artículo original

\section{SUMMARY}

This article forms part of a collaborative study in which research teams from Canada and Mexico participated. The general objective is to describe the cultural adaptation and semantic validation of three instruments for measuring stigma and mental illness in Mexico City. The criteria of understanding, acceptance, relevance and semantic integrity were used to adapt the following instruments: The Internalized Stigma Inventory (ISMI), the Opinions on Mental Illness Survey (OMI) and the Devaluation and Discrimination Scale (DDS). Four Individual interviews and four group interviews were carried out with 37 informants from different groups (health personnel, persons with diagnostic of mental disorders, relatives and the general population). Lastly, an analysis was carried out on the contents of the information obtained. The instruments proved to be culturally appropriate for the population in Mexico in the various groups studied. The adapted versions would be useful for establishing comparative analyses with other countries. The scope and limitations of the process of semantic equivalence were analyzed in the transcultural research.

Key words: Cultural adaptation, semantic validation, Opinions on Mental Illness (OMI), the Devaluation and Discrimination Scale (DDS), The Inventory of Internalized Stigma of Mental Illness (ISMI), Mexico.
Este trabajo forma parte de un estudio colaborativo en el que participan equipos de investigación en Canadá y México.

El objetivo general consiste en describir el proceso de adaptación cultural y validación semántica de tres instrumentos de medición sobre el estigma y la enfermedad mental en la Ciudad de México. A partir de los criterios de comprensión, aceptación, relevancia e integridad semántica se adaptaron los siguientes instrumentos: el Inventario de Estigma Internalizado (ISMI), el Cuestionario de Opiniones hacia la Enfermedad Mental (OMI) y la Escala de Percepción de la Devaluación y Discriminación hacia la Enfermedad Mental (DDS). Para ello se llevaron a cabo cuatro entrevistas individuales y cuatro grupales con 37 informantes de diversos sectores (personal de salud, personas con diagnóstico de trastorno mental grave, familiares y población general). Finalmente se efectuó un análisis de contenido de la información obtenida. Los instrumentos adaptados fueron culturalmente apropiados para la población de los diferentes grupos estudiados en México. Las versiones adaptadas serán de utilidad para establecer análisis comparativos con otras regiones. Se analizan los alcances y limitaciones del proceso de equivalencia semántica en la investigación transcultural.

Palabras clave: Adaptación cultural, validación semántica, Cuestionario de Opiniones hacia la Enfermedad Mental (OMI), Escala de Percepción de la Devaluación y Discriminación hacia la Enfermedad Mental, Inventario de Estigma Internalizado de la Enfermedad Mental (ISMI), Ciudad de México.

\section{INTRODUCCIÓN}

La investigación transcultural sobre los procesos de exclusión y discriminación hacia las personas afectadas por padecimientos mentales ha cobrado relevancia en la actualidad, ya que constituye un tema central relacionado con los derechos humanos y la lucha por garantizar la equidad en el acceso a la atención de la población afectada. ${ }^{1}$ Hoy en día, el propósito que se plantea es lograr incidir en las políticas públicas a ni- vel internacional para la disminución del estigma hacia dichos padecimientos, para lo cual se requieren investigaciones basadas en la evidencia que permitan fundamentar estas propuestas, de ahí el creciente interés por desarrollar estudios en poblaciones específicas (p.ej. personal de salud, personas afectadas, población general, etc.) en diferentes regiones..$^{2,3}$ Una de las principales dificultades es contar con parámetros comunes de medición que sean instrumentos culturalmente sensibles a fin de poder emplearlos en estudios comparativos. ${ }^{1}$

Dirección de Investigaciones Epidemiológicas y Psicosociales. Instituto Nacional de Psiquiatría Ramón de la Fuente Muñiz.

2 Douglas Mental Health University Institute. McGill University. Montreal, Canada.

Correspondencia: Dra. Jazmín Mora-Rios. Dirección de Investigaciones Epidemiológicas y Psicosociales. Instituto Nacional de Psiquiatría Ramón de la Fuente Muñiz. Calz. México-Xochimilco, 101, San Lorenzo Huipulco, Tlalpan, 14370, México, DF. E-mail: morarj@imp.edu.mx

Recibido primera versión: 28 de febrero de 2012. Segunda versión: 14 de mayo de 2012. Aceptado: 13 de junio de 2012. 
Este trabajo es parte de una investigación comparativa $\mathrm{y}$ transcultural en la que participan equipos de Canadá ${ }^{4} \mathrm{y}$ México, ${ }^{5}$ cuyo propósito es: "lograr avances multidisciplinarios en la investigación sobre el estigma y la dinámica de la discriminación estructural y la exclusión social hacia la enfermedad mental", considerando distintos sectores de la población, cuya fase inicial consistió en la adaptación semántica de los instrumentos empleados: a) la Escala de Percepción de la Discriminación y la Devaluación hacia la Enfermedad Mental (DDS), b) el Inventario de Estigma Internalizado de la Enfermedad Mental (ISMI) y c) el Cuestionario de Opiniones hacia la Enfermedad Mental (OMI).

Durante muchos años la adaptación se limitaba a la traducción casi literal de los instrumentos de medición para su posterior aplicación, sin prestar mayor atención a sus contenidos, lo que, sin duda, tiene importantes implicaciones en los hallazgos ${ }^{6}$ y su interpretación. Hoy en día, en el campo de la investigación transcultural la adaptación de instrumentos implica un proceso más riguroso que trasciende la traducción del idioma original de un instrumento, particularmente cuando es empleado en un contexto sociocultural y lingüísticamente distinto de aquél para el que fue creado, a fin de alcanzar la equivalencia de significado en ambos contextos. En el ámbito de la investigación en salud, ${ }^{7}$ son diversas las experiencias y específicamente en el campo de la salud mental se han utilizado estos métodos para el estudio de la psicosis, ${ }^{8}$ los trastornos de conducta alimentaria; ${ }^{9}$ los trastornos de ansiedad, la fobia social ${ }^{10}$ y la esquizofrenia, ${ }^{11}$ así como en la evaluación de la calidad de vida en personas con trastorno por déficit de atención e hiperactividad $(\mathrm{TDAH})^{12}$ y en población infantil, ${ }^{13-15}$ entre otros.

Si bien no existe un consenso respecto al procedimiento metodológico más adecuado para realizar la adaptación de los instrumentos, las estrategias grupales son un recurso comunmente empleado durante el proceso. El método Delphi, ${ }^{16}$ que consiste en una técnica de pronósticos basada en un proceso grupal con informantes clave, ha inspirado una parte considerable de la investigación. Uno de los principios en los que se fundamenta es la idea de que el juicio subjetivo de un grupo de expertos en determinado campo contribuye a un mayor conocimiento sobre ciertos temas a partir de la reflexión grupal, lo cual tiene gran alcance en contraste con el trabajo individual, aspecto crucial para la toma de decisiones. ${ }^{17}$

El método Delphi involucra una serie de etapas en las cuales se invita a un panel de expertos en un tema particular, a colaborar en el análisis y discusión a fin de lograr un cierto consenso. ${ }^{18}$ Este procedimiento es particularmente útil sobre todo al abordar temas en los cuales el conocimiento es controversial, no existe evidencia suficiente o bien son considerados como "tabú" entre la población. ${ }^{7,19}$ En ese sentido la participación grupal contribuye no solo a ampliar el conocimiento sino que ayuda a generar ideas e hipótesis nuevas. Para lograr este propósito la elección de los participantes es clave, no sólo por su amplio conocimiento en el tema sino también por el grado de disposición y apertura que muestren para compartir sus ideas con los demás. Asimismo, la diversidad de los integrantes del panel enriquece el conocimiento ya que permite la inclusión de distintas perspectivas. ${ }^{20}$

Autores como Westermeyer y Janca, ${ }^{21}$ Van Ommeren et al., ${ }^{22}$ Wild et al. ${ }^{23}$ y Eremenco et al., ${ }^{17}$ han planteado interesantes contribuciones a nivel metodológico que son de gran utilidad durante el proceso de adaptación cultural. Entre ellas destacan la reflexión sobre las discrepancias entre los instrumentos de medición y los aspectos socioculturales cuya expresión más clara podemos encontrarla en el efecto definido por Kleinman ${ }^{24}$ como "falacia categorial". Dicho concepto alude a la aplicación de una categoría nosológica desarrollada por un grupo particular y su proyección en personas de otras culturas para quienes esta categoría carece de coherencia y donde su validez no ha sido establecida. Esto da lugar a una imposición de criterios diagnósticos que terminan por ignorar el contexto social, cultural, económico y político del grupo más amplio, lo cual se postula como una de las fuentes principales de variación en el ámbito de la salud-enfermedad. ${ }^{25}$ En este sentido, la inclusión de los aspectos cualitativos contribuye no sólo a lograr una mayor comprensión del entorno de estudio, sino también a la validación de los instrumentos para recolección de datos y la interpretación de resultados. ${ }^{26,27}$ De ahí que la segunda contribución destacada a nivel metodológico sea el señalar la importancia de emplear estrategias multi-método, además del esfuerzo por desarrollar normas básicas que guíen al investigador durante todo el proceso. Otras estrategias son las evaluaciones de la calidad de los reactivos y su nivel de dificultad, de la similitud de interpretación; así como la elaboración de traducciones inversas de las versiones adaptadas al contexto local al idioma original de los instrumentos (back translation). ${ }^{15,28}$

La diversidad de enfoques y propuestas ${ }^{19,22}$ hacen de los procesos de adaptación una tarea complicada, pero a la vez necesaria para contar con análisis más rigurosos, de manera que la elección del método más adecuado para lograr la equivalencia semántica se establece a partir de los objetivos e intereses que se planteen en la investigación. Al respecto, Manson ${ }^{29}$ propone cuatro criterios fundamentales para lograr que un instrumento sea culturalmente apropiado: 1) nivel de comprensión, 2) aceptabilidad, 3) relevancia y 4) completitud ${ }^{22}$ o completeness, término originalmente planteado en idioma inglés que hace referencia a la idea de totalidad, es decir de lograr incorporar todos aquellos aspectos que dan cuenta de un fenómeno de estudio para lograr cierta integridad conceptual. Para efectos de este trabajo adoptamos este último término en idioma español, dado que se acerca más al sentido y significado del concepto original en inglés. En el cuadro 1 se muestran estos criterios.

El nivel de comprensión tiene que ver con atributos específicos como sencillez y claridad de los reactivos; un reactivo incomprensible es aquél cuyo contenido no es evidente para la población, por ejemplo, el uso de la terminología 
Cuadro 1. Criterios de análisis en la adaptación cultural y validación semántica

\begin{tabular}{|c|c|}
\hline Niveles & Descripción \\
\hline Comprensión & $\begin{array}{l}\text { - Evaluación de los contenidos que permite definir } \\
\text { qué tan entendible es el reactivo para la pobla- } \\
\text { ción a quien va dirigido. } \\
\text { Traducción libre del uso de terminología médica } \\
\text { a fin de ser más comprensible para la población. }\end{array}$ \\
\hline Aceptabilidad & $\begin{array}{l}\text { - Determina que los contenidos de los reactivos no } \\
\text { incomoden o resulten ofensivos para la población. }\end{array}$ \\
\hline Relevancia & $\begin{array}{l}\text { - Se refiere a que los reactivos deben estar rela- } \\
\text { cionados con el fenómeno o constructo que está } \\
\text { siendo medido en la cultura local. }\end{array}$ \\
\hline $\begin{array}{l}\text { Integridad } \\
\text { semántica }\end{array}$ & $\begin{array}{l}\text { - Implica la total equivalencia que debe existir en- } \\
\text { tre los contenidos de ambas versiones de los ins- } \\
\text { trumentos. }\end{array}$ \\
\hline
\end{tabular}

médica, que en muchos casos no tiene un significado claro para la población. De ahí la importancia de tomar en consideración las características socioculturales de la población objetivo. Un instrumento cumple con los criterios de aceptabilidad cuando sus contenidos son culturalmente apropiados para la población, de manera que es importante evitar el uso de lenguaje que pudiera resultar confuso u ofensivo. La relevancia tiene que ver con la importancia de que los reactivos mantengan estrecha relación con el objeto de estudio, es decir que sean útiles y pertinentes para su abordaje. Por último, la integridad conceptual o completeness, que como se mencionó anteriormente no cuenta con una traducción directa al castellano y que alude a la capacidad de integrar a la estructura del instrumento, en la medida de lo posible, de todos aquellos elementos locales que conforman el fenómeno de estudio. De no existir esta correspondencia, el instrumento no cumpliría con los niveles de equivalencia semántica, conceptual y técnica necesarios para su aplicación.

Con base en lo anterior, el objetivo de este artículo es exponer el proceso de adaptación transcultural de tres instrumentos de medición que cuentan con amplia tradición en la investigación sobre el estigma y la enfermedad mental (DDS, ISMI y OMI), ${ }^{30}$ por el interés de contar con versiones acordes al lenguaje y características socioculturales de la población mexicana, que permitan el análisis comparativo con otras poblaciones. Al final se analizan los alcances y limitaciones encontradas durante el proceso y finalmente se plantea la discusión en torno a la importancia de llevar a cabo la equivalencia semántica de instrumentos en la investigación transcultural.

\section{MATERIAL Y MÉTODOS}

Este trabajo forma parte de una investigación comparativa y transcultural más amplia en la que participan equipos de investigación de Canadá y México. Es una propuesta planteada originalmente por Pedersen (2009) cuyo objetivo global era conformar una red de investigación a fin de lograr avances multidisciplinarios en la investigación sobre el estigma y la dinámica de la discriminación estructural y la exclusión social hacia la enfermedad mental. ${ }^{4}$ México se suma a este interés y desarrolla un estudio similar empleando los mismos instrumentos de medición que se aplicaron en Canadá. Una vez que se obtiene el financiamiento, se pone en marcha este proyecto.

Esta investigación fue evaluada por el Comité de Ética del Instituto Nacional de Psiquiatría Ramón de la Fuente Muñiz. En todo el proceso se emplearon formatos de consentimiento informado, además de la autorización de los informantes para el registro en audio de las entrevistas.

En la figura 1 se muestra el procedimiento metodológico que se siguió para la adaptación de los instrumentos.

\section{Procedimiento para la adaptación cultural}

El proceso se inicia con el envío de las versiones originales en idioma inglés de los instrumentos por un equipo de investigadores canadienses del Douglas Hospital Research Centre, en Montreal. El primer paso fue la traducción al idioma español de cada uno de los cuestionarios, la cual estuvo a cargo de una especialista de nacionalidad inglesa con amplia experiencia en la investigación psicosocial.

Los cuestionarios incluidos en el análisis fueron los siguientes:

a) La Escala de Percepción de la Discriminación y la Devaluación hacia la Enfermedad Mental (Devaluation Discrimination Scale, DDS). ${ }^{31}$ Se compone de doce reactivos, cuyas respuestas están en función de lo que "la mayoría de la gente cree o piensa", a fin de reducir la deseabilidad social. El formato de respuesta es una escala Likert de cuatro puntos que va de $0=$ totalmente en desacuerdo a $3=$ totalmente de acuerdo. El instrumento tiene una consistencia interna global de 0.76 (alpha de Cronbach).

b) Inventario de Estigma Internalizado de la Enfermedad Mental (Internalized Stigma of Mental Illness Inventory, ISMI). ${ }^{32}$ Los autores del instrumento retoman la definición de Corrigan (1998) de estigma internalizado como "la devaluación, vergüenza, secrecía y retraimiento consecuencia de aplicar los estereotipos negativos a sí mismo", 32 y plantean un cuestionario de 29 reactivos que mide cinco grandes áreas: a) alienación, b) adhesión al estereotipo, c) experiencia de discriminación, d) retraimiento social y e) resistencia al estigma. El formato de respuesta es una escala Likert de cuatro puntos $(0=$ totalmente en desacuerdo a $3=$ totalmente de acuerdo). La consistencia interna es adecuada (alpha=0.90).

c) Cuestionario de Opiniones acerca de la Enfermedad Mental (Opinions about Mental Illness Scale, OMI). ${ }^{33}$ Fue desarrollado originalmente por Cohen y Struening en la década de 1960. Consta de 51 reactivos que evalúan cinco dimensiones: 1) autoritarismo, 2) benevolencia, 3) ideo- 


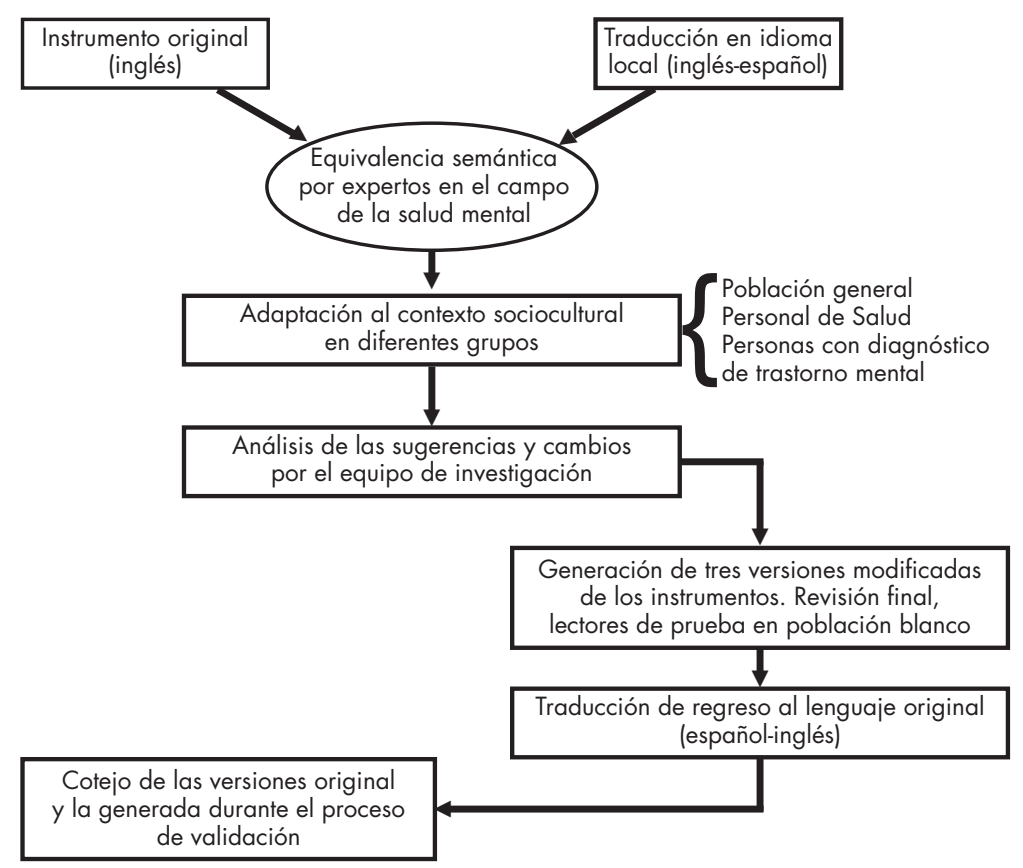

Figura 1. Estrategia metodológica para la adaptación cultural de los instrumentos.

logía sobre higiene mental, 4) restrictividad social y 5) etiología interpersonal. El rango de respuesta va de $0=$ totalmente en desacuerdo a $5=$ totalmente de acuerdo. La consistencia interna de la prueba es de 0.83 .

\section{Panel de expertos}

Se organizó un grupo de trabajo con profesionales provenientes de diversas disciplinas que cumplieran con las siguientes características: 1) contar con amplia experiencia en el campo de la salud mental y 2) ser preferentemente bilingües. El grupo quedó conformado por ocho integrantes, tres psicólogas, tres psiquiatras, uno de ellos de sexo femenino; una trabajadora social y un antropólogo investigador. A excepción de dos investigadores y el antropólogo, todos laboraban en centros de atención especializada. Una vez que aceptaron participar en el estudio se les entregó una copia de las versiones en inglés y en español de los instrumentos, con el propósito de que leyeran y corroboran la correspondencia semántica entre ellas para posteriormente compartir sus observaciones y puntos de vista con otros expertos en el área de salud mental, con relación a los contenidos.

La entrevista grupal tuvo una duración de dos horas y media, se efectuó en una sesión y se llevó a cabo en las instalaciones del centro de atención. En la sesión participaron dos psicólogas del equipo de investigación, una de ellas fungió como coordinadora y la otra como observadora. El registro de la información se realizó por medio de notas y grabación en audio de las entrevistas, previa autorización de los participantes.

Los tópicos de estudio fueron los siguientes:

1) La adecuación de las versiones de los instrumentos del inglés al español;

2) Comprensión de los contenidos;

3) Claridad en los reactivos;

4) Relevancia y pertinencia de los reactivos para evaluar estigma;

5) Opiniones sobre los reactivos, si pueden resultar ofensivos o que atentan contra la integridad de los informantes;

6) Reactivos importantes para evaluar estigma que no fueron incluidos en los instrumentos;

7) Observaciones, sugerencias de los informantes que pueden ser de utilidad para el estudio y comentarios finales.

Una vez que se obtuvieron las versiones de los tres instrumentos revisadas previamente por el grupo de expertos, se procedió a la validación de sus contenidos dependiendo de la población a la cual iban dirigidos. Se organizaron dos grupos de trabajo en la población general para analizar los contenidos del DDS; uno de ellos estuvo integrado por familiares de personas afectadas por un padecimiento mental. Para analizar los contenidos del ISMI se llevaron a cabo dos entrevistas individuales con personas diagnosticadas con un trastorno mental grave; en tanto que para el OMI se realizaron dos entrevistas individuales con profesionales de la salud. A continuación se describen las características de los integrantes en la población objetivo.

a) Familiares de personas que han sido diagnosticadas con un padecimiento mental. Se organizó una entrevista grupal en la que participaron quince integrantes de una asociación civil quienes colaboraron voluntariamente en la investigación e incluso brindaron sus instalaciones para realizar la entrevista. El contacto fue a través de un miembro de dicha asociación. Aunque se tenía contemplada la parti- 
cipación de un menor número de integrantes, durante la dinámica grupal se fueron incorporando otras personas. Pese a que hubo algunas dificultades para escuchar las opiniones de todos los participantes, la entrevista se desarrolló adecuadamente y se hicieron aportaciones valiosas con relación a los contenidos del instrumento. Tuvo una duración de una hora aproximadamente y, al final, se agradeció la colaboración de los familiares estableciendo el contacto para subsecuentes fases de la investigación.

b) Población general. Se convocó a los integrantes de una asociación civil para llevar a cabo la entrevista grupal, al final sólo asistieron cuatro personas, dos hombres y dos mujeres de entre los 37 y 55 años. Tres de ellos brindaban servicios comunitarios en dicha asociación y la otra persona, pertenecía al área administrativa de una institución pública.

c) Personas que presentan un diagnóstico psiquiátrico. Se llevaron a cabo dos entrevistas individuales con lectores de prueba; ambos con diagnóstico de trastorno mental grave y de sexo masculino, quienes fueron contactados en la institución donde recibían atención. Además de contar con un diagnóstico de trastorno mental grave, otros criterios de inclusión fueron que estuvieran en tratamiento ambulatorio y que aceptaran participar en el estudio. Las entrevistas tuvieron una duración de 50 minutos.

d) Profesionales de la salud. Se convocó a una psicóloga y un psiquiatra para participar como lectores de prueba a través de dos entrevistas individuales. Ambos contaban con amplia experiencia en la salud mental, uno en el campo de la investigación y el otro en la atención. Las entrevistas tuvieron una duración promedio de una hora y media.

Finalmente, todo el proceso de traducción y adaptación tuvo lugar en el transcurso de tres meses durante los cuales se promovió la discusión y reflexión sobre aspectos controversiales, por medio del planteamiento de preguntas abiertas, profundizando en los significados, buscando incorporar los diferentes puntos de vista de los participantes acerca de los contenidos de los cuestionarios.

\section{Análisis de la información}

La información obtenida a partir de las entrevistas individuales y de los grupos de trabajo fue registrada en audio y transcrita posteriormente para facilitar el análisis del contenido. Se organizaron las observaciones y comentarios por tipo de población entrevistada, tomando como referencia los criterios propuestos por Manson ${ }^{30}$ y Van Ommeren et al. ${ }^{22}$ para la adaptación cultural, en términos de: 1) comprensión, 2) aceptación, 3) relevancia y 4) integridad semántica.

\section{RESULTADOS}

En los cuadros 2 y 3 se muestran los principales hallazgos con base en los criterios anteriormente descritos para lograr la equivalencia semántica de los instrumentos.

1) La Escala de Percepción de la Discriminación y la Devaluación hacia la Enfermedad Mental (DDS).

a) Comprensión. Las principales observaciones tuvieron que ver con la extensión de los reactivos (p.ej., " $L a$ mayoría de la gente aceptaría como maestra de niños en una escuela, a alguien que se ha recuperado completamente de una enfermedad mental severa"), por lo que fueron considerados como poco claros y confusos. Otros más, incluían negación en los contenidos: "La mayoría de la gente no contrataría para cuidar a sus hijos a alguien que ha estado hospitalizado debido a una enfermedad mental grave, aunque esta persona ya se encuentre recuperada" o "La mayoría de los trabajos no considerarían la solicitud de empleo de alguien que ha estado hospitalizado debido a una enfermedad mental". Otros reactivos fueron considerados como ambiguos (p.ej., "La mayoría de la gente piensa que un enfermo mental tiende a ser violento" y "La mayoría de los familiares de un enfermo mental se avergüenzan de él"), por lo que era difícil brindar una respuesta, la cual estaba en función de aspectos más específicos como tipo de padecimiento, nivel de gravedad, así como de los recursos de apoyo social.

b) Aceptación. En términos generales los informantes coincidieron en que los contenidos del DDS eran adecuados para la población. Sin embargo, el hecho de que los reactivos del instrumento estén planteados en términos de "lo que la mayoría de la gente piensa", a fin de disminuir los efectos de la deseabilidad social, generó incomodidad entre los participantes, ya que consideraron que su opinión personal era dejada al margen.

c) Relevancia. Los informantes estuvieron de acuerdo en la pertinencia de los reactivos del DDS, aunque consideraron que eran insuficientes para abordar el tema de estudio por lo que era necesario explorar diferentes ámbitos a nivel laboral, familiar, escolar, comunitario y de pareja. Para cubrir estas ausencias sugirieron la inclusión de veintiún reactivos, los cuales se sometieron a un análisis para elegir aquellos que resultaban pertinentes por su cercanía con el objeto de estudio. Los reactivos seleccionados se presentan en el cuadro 3.

2) Inventario de Estigma Internalizado de la Enfermedad Mental (ISMI).

a) Comprensión. En este rubro se identificaron ciertos problemas en la comprensión de los reactivos que incluían doble negación, por ejemplo, "Trato de no acercarme a la gente que no tiene una enfermedad mental para evitar ser rechazado". Algunos otros al incluir más de una idea resultaban muy extensos (p.ej. "No tengo tanta vida social como antes, porque mi enfermedad mental puede hacerme ver mal o que me comporte de manera "extraña»"). Por otro lado, se observó cierta ambigüedad en algunos reactivos como: "La gente que padece enfermedades mentales tiende a ser violenta", "La gente con enfermedades mentales no debería casarse" y "La gente con enfermedades mentales 
Cuadro 2. Observaciones a los instrumentos durante el proceso de adaptación cultural y validación semántica

\begin{tabular}{|c|c|c|c|}
\hline & ISMI & DDS & OMI \\
\hline Comprensión & $\begin{array}{l}\text { - Extensión de los reactivos (unión de dos } \\
\text { distintas ideas en una misma oración). } \\
\text { - Ambigüedad de ciertos términos y ne- } \\
\text { cesidad de precisar aspectos asociados } \\
\text { a los contenidos de algunos reactivos } \\
\text { (contextualización). } \\
\text { - Evitar el uso de negación en los reacti- } \\
\text { vos. }\end{array}$ & $\begin{array}{l}\text { - Extensión de los reactivos (unión de dos } \\
\text { distintas ideas en una misma oración). } \\
\text { - Ambigüedad de ciertos términos y ne- } \\
\text { cesidad de precisar aspectos asociados } \\
\text { a los contenidos de algunos reactivos } \\
\text { (contextualización). } \\
\text { - Evitar el uso de negación en los reacti- } \\
\text { vos. }\end{array}$ & $\begin{array}{l}\text { - Extensión de los reactivos (unión } \\
\text { de dos distintas ideas en una mis- } \\
\text { ma oración). } \\
\text { - Ambigüedad de ciertos términos } \\
\text { y necesidad de precisar aspectos } \\
\text { asociados a los contenidos de } \\
\text { algunos reactivos (contextualiza- } \\
\text { ción). }\end{array}$ \\
\hline Aceptación & $\begin{array}{l}\text { - La aplicación y uso reiterado del térmi- } \\
\text { no enfermedad mental fue considerado } \\
\text { ofensivo o generador de incomodidad } \\
\text { en informantes que no estaban de } \\
\text { acuerdo con el diagnóstico médico. } \\
\text { - Reactivos referentes al matrimonio o } \\
\text { sentimientos de inferioridad y vergüen- } \\
\text { za fueron considerados por algunos } \\
\text { informantes como ofensivos. }\end{array}$ & $\begin{array}{l}\text { - Responder de acuerdo con la opinión } \\
\text { de la mayoría de la gente generó inco- } \\
\text { modidad y confusión. Se sugirió como } \\
\text { más adecuado indagar acerca de la } \\
\text { propia opinión del informante. }\end{array}$ & $\begin{array}{l}\text { - Los contenidos fueron conside- } \\
\text { rados aceptables para la pobla- } \\
\text { ción a quien se dirige. } \\
\text { - No se identificaron reactivos } \\
\text { ofensivos. }\end{array}$ \\
\hline Relevancia & $\begin{array}{l}\text { - Sus contenidos se consideraron adecua- } \\
\text { dos, relevantes y relacionados con el } \\
\text { tema de estudio. } \\
\text { - Se incluyeron } 5 \text { reactivos por conside- } \\
\text { rarse convenientes a la comprensión del } \\
\text { tema de estudio. }\end{array}$ & $\begin{array}{l}\text { - Sus contenidos se consideraron adecua- } \\
\text { dos, relevantes y relacionados con el } \\
\text { fenómeno de estudio. } \\
\text { - Se incluyeron } 7 \text { reactivos por conside- } \\
\text { rarse convenientes a la comprensión del } \\
\text { tema de estudio. }\end{array}$ & $\begin{array}{l}\text { - Sus contenidos se consideraron } \\
\text { adecuados, relevantes y relaciona- } \\
\text { dos con el fenómeno de estudio. } \\
\text { - Actualización de los términos uti- } \\
\text { lizados. } \\
\text { - Se incluyeron } 8 \text { reactivos por } \\
\text { considerarse convenientes a la } \\
\text { comprensión del tema de estudio. }\end{array}$ \\
\hline $\begin{array}{l}\text { Integridad } \\
\text { semántica }\end{array}$ & \multicolumn{3}{|c|}{$\begin{array}{l}\text { - Las versiones traducidas y adaptadas al español de los tres instrumentos (ISMI, DDS y OMI) se sometieron a un análisis por } \\
\text { parte del equipo de investigación en Canadá a fin de lograr el nivel adecuado de equivalencia semántica con relación a } \\
\text { los instrumentos originales. }\end{array}$} \\
\hline
\end{tabular}

no puede vivir una vida gratificante y plena", pues según los lectores de prueba, esto tiene que ver con el tipo y nivel de gravedad del padecimiento, además del contexto social y familiar por lo que resulta difícil generalizar una respuesta. No obstante, en términos generales el instrumento fue comprensible para la población.

b) Aceptación. El principal señalamiento que hubo a este instrumento tiene que ver con el nivel de conciencia de enfermedad de la persona entrevistada. En ese sentido, el término "enfermedad mental" puede resultar ofensivo en aquellos casos en que el informante no está de acuerdo con el diagnóstico médico, de manera que el escucharlo reiteradamente genera incomodidad: "Me apena y me avergüenza tener una enfermedad mental", "Me siento fuera de lugar en el mundo porque tengo una enfermedad mental", "Me siento inferior a la gente que no tiene enfermedades mentales" y "No puedo aportarle nada a la sociedad por tener una enfermedad mental". Al respecto hubo controversia con otros informantes quienes no los consideraron ofensivos.

c) Relevancia. Si bien en términos generales los participantes consideraron pertinentes los reactivos contenidos en el ISMI, el panel de expertos y los lectores de prueba sugirieron la inclusión de quince reactivos adi- cionales, los cuales fueron sometidos a un análisis por parte del equipo de investigación identificando aquellos en los cuales había consenso y que eran útiles para explorar estos temas. Se eligieron cinco reactivos que fueron incluidos en la versión final del instrumento y que se muestran en el cuadro 3.

3) Cuestionario de Opiniones hacia la Enfermedad Mental $(O M I)$.

a) Comprensión. En general, de acuerdo con el punto de vista de los informantes, los contenidos del instrumento cumplieron con este criterio, están planteados en un lenguaje sencillo y comprensible para la población objetivo. Al igual que en los instrumentos anteriores, las principales observaciones fueron con relación a la ambigüedad (p.ej. "La mayoría de la gente que alguna vez estuvo en servicios psiquiátricos puede ser confiable para cuidar niños", "A todos los pacientes que ingresan a los servicios de psiquiatría se les debería hacer una operación indolora para prevenir que tengan hijos"), cuyas respuestas están en función de otros factores que requieren mayor contextualización para brindar una respuesta concreta. El reactivo acerca de "Si los hijos de padres con enfermedades mentales fueran criados por padres normales, probablemente no se volverían enfermos mentales" fue considerado muy 
Cuadro 3. Reactivos incorporados a los instrumentos durante la adaptación cultural

Inventario de Estigma Internalizado de la Enfermedad Mental (ISMI)

30. Merezco una mayor consideración de los demás por tener una enfermedad mental.

31. A partir de mi enfermedad mental, me siento más unido a mi familia.

32. Tener una enfermedad mental no es un obstáculo para que tenga una relación de pareja.

33. La mayor parte de mis problemas se resolverían si no tuviera una enfermedad mental.

34. Tener una enfermedad mental hace que vea el futuro con mucha incertidumbre.

Escala de Percepción de la Discriminación y la Devaluación hacia la enfermedad mental (DDS)

13. La mayoría de la gente piensa que una mujer es más propensa a las enfermedades mentales.

14. La mayoría de la gente piensa que un enfermo mental tiende a ser violento.

15. La mayoría de la gente siente miedo al estar frente a un enfermo mental.

16. La mayoría de la gente piensa que las personas que tienen una enfermedad mental son débiles de carácter.

17. La mayoría de los familiares de un enfermo mental se avergüenzan de él.

18. La mayoría de las escuelas no aceptarían a una persona que ha estado hospitalizada por una enfermedad mental.

19. La mayoría de la gente piensa que las personas que tienen una enfermedad mental están pagando un castigo por algo que hicieron.

Cuestionario de Opiniones acerca de la Enfermedad Mental (OMI)

52. Los hospitales psiquiátricos deberían ubicarse en zonas lo más alejadas posible de la población general.

53. Me siento tranquilo al estar cerca de una persona que tiene alguna enfermedad mental severa.

54. Las personas que se masturban en exceso, tienen mayor probabilidad de tener una enfermedad mental.

55. Las mujeres son más propensas a desarrollar una enfermedad mental.

56. Mantener contacto frecuente con las personas que tienen algún trastorno mental severo puede aumentar el riesgo de desarrollar una enfermedad mental.

57. Los profesionales que laboran en el área de salud mental son más propensos a desarrollar una enfermedad mental.

58. A los psiquiatras y psicólogos que tienen una enfermedad mental severa y que ya están recuperados, se les debería prohibir atender a otros pacientes psiquiátricos.

59. En muchos casos, la única manera de tratar a los pacientes psiquiátricos es con mano dura.

extenso lo que puede generar confusión. Asimismo, los participantes sugirieron precisar más ciertos conceptos como "gente normal", "malos pensamientos", "enfermedad mental grave", "mayor privacidad" y "enfermedad mental" que resultaban ambiguos.

b) Aceptación. En general, el OMI cumplió con este criterio ya que ninguno de los reactivos fue considerado por los informantes como inapropiado $\mathrm{u}$ ofensivo para la población.

c) Relevancia. Aun cuando este cuestionario fue calificado como extenso por parte de los informantes, consideraron que los contenidos eran pertinentes ya que abarcaban distintas áreas relacionadas con el tema de estudio, como las actitudes y creencias vinculadas con la enfermedad mental que escuchan con frecuencia durante su quehacer profesional y que "están muy relacionadas con el contexto social en que vivimos", como lo refirió uno de los participantes.

Otra observación que se planteó fue la necesidad de actualizar ciertos términos, debido a que los contenidos de algunos de ellos fueron creados por sus autores durante la década de los años 1960 en el que predominaba la institucionalización de los pacientes psiquiátricos, de manera que hoy en día tienen un sentido distinto. Por ejemplo, el uso del término "pabellón", que si bien se mantiene en algunas instituciones en México, en muchas otras ha caído en desuso. Como resultado del ejercicio de reflexión grupal, de los veinticuatro reactivos propuestos inicialmente sólo se eligieron ocho considerados como los más importantes y que se muestran en el cuadro 3.

\section{Integridad conceptual}

Las tres versiones adaptadas fueron enviadas al equipo de investigación en Canadá, el cual analizó las similitudes y discrepancias a fin de establecer el nivel de equivalencia semántica obtenida en México con respecto a los instrumentos originales. Por último, se estableció un consenso entre los equipos de investigación en ambos países acerca de los cambios que serían incorporados antes de la aplicación en el estudio definitivo y facilitar con ello la comparación transcultural.

Durante el análisis comparativo se observaron discrepancias en seis reactivos considerando a los tres instrumentos, por lo que fueron modificados con la finalidad de alcanzar la equivalencia semántica requerida. Asimismo, se efectuaron otros cambios en los tiempos verbales empleados, hubo mayor precisión en algunos términos (p.ej. servicios psiquiátricos en lugar de pabellón, haciendo referencia a otros contextos como los servicios que se ofrecen en hospitales generales). Además de llevar a cabo algunas precisiones respecto de las instrucciones, se mantuvieron las categorías de respuesta, modificando únicamente la redacción (p.ej. "totalmente de acuerdo" en lugar de "fuertemente de acuerdo"). Cabe señalar que surgieron otras propuestas que al final no pudieron ser incorporadas ya sea porque se alejaban del objeto de estudio o bien porque implicaban cambios mayores en la estructura de los instrumentos, lo que de al- 
gún modo podría obstaculizar la comparación transcultural. El proceso finalizó con la traducción de la versión adaptada semánticamente del idioma español al inglés.

\section{DISCUSIÓN}

La principal contribución de este trabajo fue la obtención de tres instrumentos que cuentan con amplia tradición en la investigación internacional sobre el estigma hacia la enfermedad mental, los cuales fueron adaptados para su aplicación entre la población de la Ciudad de México, lo que permitirá establecer el análisis comparativo con otra investigación que se realiza de manera simultánea en Canadá, lo que constituye una aportación valiosa frente al desafío que se ha planteado en la investigación en esa línea que consiste en contar con parámetros comunes para la medición del estigma. ${ }^{1}$

En términos generales, los instrumentos considerados en esta investigación cumplen con los criterios de comprensión, aceptación, relevancia e integridad semántica. ${ }^{22,29}$ No obstante, las principales observaciones que se plantearon durante el proceso tienen que ver con el criterio de comprensión, en tres aspectos básicos: ambigüedad, extensión y redacción de los reactivos. Respecto al criterio de aceptación, los lectores de prueba consideraron que el ISMI, dirigido a personas con un padecimiento mental, podría resultar ofensivo para quienes no están de acuerdo con el diagnóstico médico o que se encuentran en un proceso inicial de búsqueda de atención, por las constantes referencias al término enfermedad mental, que en un momento dado podrían incomodarles. En el caso del OMI y el DDS no hubo otras sugerencias relevantes.

Con relación al criterio de relevancia, destacan, por un lado, la importancia de actualizar los contenidos del OMI, aspecto al cual se han referido otros autores, ${ }^{34}$ ya que algunos reactivos de la versión original incluyen términos en desuso dentro de la práctica médica en México (p.ej. pabellón, guardias) o que se limitaban a los servicios psiquiátricos especializados, dejando al margen la atención psiquiátrica que se ofrece en hospitales generales; en tanto que para el DDS hubo un mayor número de propuestas para la inclusión de nuevos reactivos, lo que indica que si bien los contenidos originales son relevantes, resultan al mismo tiempo insuficientes para cubrir otros aspectos considerados como importantes para el abordaje del tema en México.

Por último, el envío de los tres instrumentos al equipo de investigadores en Canadá a fin de cumplir con el criterio de integridad semántica, permitió incorporar cambios necesarios para contar con versiones equivalentes en ambos países. Asimismo, se incluyeron otros reactivos propuestos específicamente para el abordaje del tema en México, con la intención de ser analizados de manera independiente por la relevancia que tienen, lo que posibilitó identificar los alcances y limitaciones de dichas mediciones con relación al obje- to de estudio a nivel local. Dentro del campo de la salud, la traducción ha sido señalada por diversos autores como una molestia o problema técnico cuya solución se alcanza mediante la incursión de otros especialistas como los traductores, quienes pese a su capacidad para referir conceptos o términos de la cultura local usualmente no se especializan en los temas de investigación, de manera que sus respuestas no pueden aplicarse de manera generalizada. ${ }^{24,25,27}$ No obstante, la mayoría de las veces se deja de lado el papel que los aspectos socioculturales juegan en dicho proceso. Las realidades locales se ven reducidas en importancia y la cultura queda desplazada en su papel de sistema que modela la experiencia y emocionalidad humanas ${ }^{25}$ situando el reto del abordaje de la diversidad cultural en la investigación en salud. De acuerdo con Sperber ${ }^{27}$ toda investigación que busca la descripción o comparación de las diferencias culturales entre poblaciones mediante el empleo de instrumentos de medición, está obligada a que éstos sean sometidos a un riguroso proceso de traducción y validación semántica. No obstante, promover el diálogo entre las interpretaciones y la reflexión sobre la propia cultura que implica el proceso de equivalencia semántica, es un esfuerzo que vale la pena implementar, aun cuando el propósito sea distinto al del análisis transcultural, ya que permite optimizar recursos y brindar mayor atención al sentido y significado que los temas de estudio tienen para la población objetivo. En esto coincidimos con autores como Kachani et al. ${ }^{9}$ quienes plantean que el proceso de adaptación semántica va más allá de la simple traducción del idioma y es parte fundamental en el proceso de investigación, particularmente en aquellos estudios que involucran mediciones estructuradas.

Respecto a la elección de las estrategias más adecuadas para la adaptación, tal y como se ha encontrado en la bibliografía, no existe un consenso, existen por el contrario diversos enfoques y estrategias de manera que es el investigador quien decide cuál es el más adecuado, dependiendo de los alcances y objetivos de su investigación, así como de los recursos con los que cuenta. Los criterios propuestos por Manson ${ }^{29}$ y Van Ommeren et al..$^{22}$ fueron de gran utilidad para el proceso de adaptación que se siguió en este trabajo, particularmente el criterio de comprensión ya que el énfasis se coloca en el sentido y significado que los contenidos de los instrumentos tienen para la población, lo cual garantiza una mayor equivalencia de significado, más allá de limitarse a la traducción literal de los términos.

Otra ventaja de emplear este recurso, es la posibilidad de considerar a los informantes no sólo como sujetos de respuesta sino integrarlos en la toma de decisiones, para lo cual las estrategias metodológicas de naturaleza cualitativa son cruciales en la identificación de temas relevantes en grupos específicos, así como para generar propuestas e ideas interesantes desde los mismos participantes. Por ejemplo, destacó entre los familiares que participaron en el estudio su preocupación por lograr cambios en la legislación, en la cobertura 
en servicios médicos y a nivel laboral. Del mismo modo, las principales inquietudes narradas por quienes presentaban una enfermedad mental fueron en relación a la estabilidad laboral, dificultades para encontrar pareja y en la sexualidad. La población general expresó su interés por aspectos referentes al proceso de salud-enfermedad mental pues consideraron que es un tema del que se habla poco y existe escasa orientación a nivel comunitario. Asimismo manifestaron que el participar en la actividad grupal representó una oportunidad de diálogo para poder externar no sólo sus dudas u opiniones, sino también para compartir su experiencia. En ese sentido las implicaciones van más allá de los aspectos metodológicos. El trabajo de Flores et al. (2011) constituye un buen ejemplo de la importancia que tiene incorporar la experiencia vivida de los grupos que viven en contextos de adversidad en la intervención psicosocial y comunitaria. ${ }^{35}$

Algunos autores señalan la utilidad del empleo de estrategias participativas como son las entrevistas grupales, para explorar temas e identificar conceptos fundamentales en la investigación. ${ }^{27}$ A este respecto cabe destacar como uno de los alcances de la utilización de esta estrategia dentro del presente estudio, la posibilidad de incluir otros temas que no se habían considerado inicialmente y que eran teóricamente relevantes desde el punto de vista de los diferentes participantes, descartando otros menos pertinentes. De igual manera, otro aspecto igualmente valioso fue el propiciar un encuentro para el análisis conjunto alrededor de los temas de estudio, ${ }^{36}$ lo que coincide con lo postulado por Knudsen et al. ${ }^{11}$ quienes colocan a los grupos focales como una estrategia adecuada para el abordaje de problemas, de conceptos, estructura y traducción de instrumentos, en la medida que permite ajustarlos con base en la reflexión grupal. En la medida en que se logre recuperar la diversidad de temas, conceptos, sectores de la población, enfoques y perspectivas será posible enriquecer más los hallazgos en torno a la equivalencia semántica. ${ }^{29}$

Por último, coincidimos con autores como Eremenco et al. ${ }^{17,26}$ quienes plantean la importancia de combinar los enfoques cualitativos y cuantitativos en la evaluación de la traducción y equivalencia de los contenidos de un instrumento. Si bien la estrategia que se siguió en México durante la adaptación cultural de los instrumentos fue esencialmente cualitativa, con lo cual fue posible contar con una visión más amplia con relación al estigma, lo cierto es que este proceso no sustituye de ningún modo a la validez de constructo de dichos instrumentos. En ese sentido se pretende dar continuidad a este trabajo y poder realizar análisis multivariados en los distintos sectores de la población estudiada.

\section{Limitaciones}

La adaptación semántica de instrumentos es de gran utilidad para el análisis de la pertinencia de los contenidos en investigaciones transculturales. Si bien es un proceso arduo, es fundamental tomar en consideración aspectos relacionados con el sentido y significado de los temas de estudio en poblaciones específicas. Sin embargo, es importante considerar algunas dificultades que pueden presentarse durante el proceso. Por ejemplo, la organización de los grupos de trabajo implica inversión de recursos humanos y materiales para llevarlos a cabo. Tampoco es sencillo el incorporar todas las observaciones de los participantes y el establecer un consenso, sobre todo en temas que generan controversia o donde hay perspectivas encontradas. Como señalan Powell ${ }^{20}$ y Boulkedid et al., ${ }^{37}$ el establecimiento del consenso es un aspecto fundamental para fortalecer la validez de los hallazgos.

Durante el proceso puede ocurrir que surjan aportaciones interesantes que finalmente no son incluidas. Por ejemplo, uno de los informantes señalaba la conveniencia de modificar el formato de respuesta del ISMI, pues más que mostrar un cierto acuerdo o no con los contenidos, debiera basarse en términos de frecuencia, que captura en mayor medida el sentido de las preguntas a nivel de proceso. Por ejemplo, en el caso de las creencias favorables este informante planteaba que al inicio de su proceso tenía creencias desfavorables hacia la enfermedad que posteriormente se fueron modificando con el tiempo. En suma, el distinguir entre las observaciones pertinentes de otras que no lo son, puede resultar complicado. No obstante, una solución posible sería optar por el análisis cualitativo en conjunto por parte del equipo de investigación, lo que facilitaría en gran medida la toma de decisiones, evitando en la medida de lo posible caer en riesgos metodológicos que pueden desviar el principal objetivo del proceso de adaptación que consiste en generar instrumentos culturalmente apropiados para la investigación comparativa y transcultural.

\section{Implicaciones en la política, programas $y$ acciones de salud mental}

Como parte de las acciones que se pretenden desarrollar a futuro se encuentra la construcción de una propuesta de intervención dirigida a la reducción del estigma hacia la enfermedad mental a nivel clínico y comunitario, que incluya a los principales actores vinculados en la problemática, empleando estrategias de carácter más cualitativo y enfoques centrados en recuperar las experiencias de los diferentes actores sociales.

\section{AGRADECIMIENTOS}

Esta investigación se llevó a cabo en cercana colaboración con el doctor Duncan Pedersen, del Institut Universitaire en Santé Mentale Douglas, Montreal, Québec; además del apoyo de la doctora Maria Elena Medina-Mora quien impulsó esta propuesta. A los Fondos Sectoriales de Investigación en Salud-CONACYT (069261) quienes brindaron el financiamiento para poner en marcha la investigación. Asimismo nuestro mayor reconocimiento va hacia todas las personas que hicieron posible el estudio, a los informantes, además del equipo de investigación, especialmente a la doctora Reyna Gutiérrez Reynaga, quien participó en la primera fase del estudio. 


\section{REFERENCIAS}

1. López M, Laviana M, Fernández L, López A et al. La lucha contra el estigma y la discriminación en salud mental. Una estrategia compleja basada en la información disponible. Rev Asoc Esp Neuropsiq 2008;28(101):43-83.

2. Acuña C, Bolis M. La estigmatización y el acceso a la atención de salud en América Latina: Amenazas y perspectivas. París, Francia: $2^{\circ}$ Organización Panamericana de la Salud. Organización Mundial de la Salud; 4-8 julio, 2005.

3. Brohan E, Elgie R, Sartorius N, Thornicroft G et al. Self-stigma, empowerment and perceived discrimination among people with schizophrenia in 14 European countries: The GAMIAN-Europe study. Schizophr Res 2010;122(1):232-238.

4. Pedersen D. Estigma y exclusión social en la enfermedad mental: apuntes para el análisis e investigación. Acta Psiquiát Psicol Am Lat 2009;55(1):39-50.

5. Mora-Ríos J, Medina-Mora ME, Natera G. Estigma y enfermedad mental. Desarrollo de un programa de intervención para su aplicación en población general y clínica. Proyecto financiado por los Fondos Sectoriales de Investigación en Salud-CONACYT (069261).

6. Arribas A. Adaptación transcultural de instrumentos. Guía para el proceso de validación de instrumentos tipo encuestas. Rev Asoc Med Bahía Blanca 2006;16(3):74-82.

7. Jiménez J. El uso de grupos focales para la adaptación cultural de un instrumento de estigma sentido en personas que viven con VIH en Puerto Rico. Tercer Congreso Iberoamericano de Investigación Cualitativa en Salud. Puerto Rico: 6-9 mayo, 2008.

8. Ros-Morente A, Vilagra-Ruiz R, Rodríguez-Hansen G, Wigman JH et al. Proceso de adaptación al castellano de la Escala de Evaluación Comunitaria de Experiencias Psíquicas (CAPE). Actas Esp Psiquiatr 2011;39(2):95-105.

9. Kachani AT, Rodrigues Barbosa AL, Brasiliano S, Cordás TA et al. Tradução, adaptação transcultural para o português (Brasil) e validação de conteúdo da Body Checking Cognitions Scale (BCCS). Rev Psiq Clín 2011;38(1):13-18.

10. Silva ACO, Nardi AE. Versão brasileira do Social Interaction Self-Statement Test (SISST): tradução e adaptação transcultural. Rev Psiq Clín 2010;37(5):199-205.

11. Knudsen HC, Vazquéz-Barquero JL, Welcher B, Gaite L et al. Translation and cross-cultural adaptation of outcome measurements for schizophrenia. EPSILON Study 2. Br J Psychiatry 2000;177(39)(Supl):s8-s14.

12. Mattos $P$, Segenreich D, Macedo Días G, Saboya E et al. Semantic validation of the Portuguese version of the Adult Attention-Deficit Disorder/ Hyperactivity Disorder (ADHD) Quality of Life Questionnaire (AAQoL) Rev Psiq Clín 2011;38(3):87-90.

13. Fernández G, Dallo MA, Durán C, Caperchione F et al. Cuestionario sobre calidad de vida pediátrica (PedsQL) versión 4.0: fase inicial de la adaptación transcultural para Uruaguay. Arch Pediatr Urug 2010;81(2):91-99.

14. Rajmil L, Serra-Sutton V, Fernández-López JA, Berra $S$ et al. Versión española del cuestionario alemán de calidad de vida relacionada con la salud en población infantil y de adolescentes. An Pediatr (Barc) 2004;60(6):514-521.

15. Berra S, Bustingorry V, Henze C, Díaz P et al. Adaptación transcultural del cuestionario kidscreen para medir calidad de vida relacionada con la salud en población argentina de 8 a 18 años. Arch Argent Pediatr 2009;107(4):307-314.

16. Farmer E. A delphi study of research priorities in Tech Prep. J Vocat Tech Educ 1998;15(1):42-49.

17. Eremenco SL, Cella D, Arnold BJ. A comprehensive method for the translation cross-cultural validation of health status questionnaires. Eval Health Prof 2005;28(2):212-232.
18. Iljaž RJ, Meglič M, Švab I. Building consensus about eHealth in Slovene primary health care: Delphi study. BMC Med Inform Decis Mak 2011;11(25):11-25.

19. Moraes CL, Reichenheim ME. Cross-cultural measurement equivalence of the Revised Conflict Tactics Scales (CTS2) Portuguese version used to identify violence within couples. Cad Saúde Pública 2002;18(3):783-796.

20. Powell C. The Delphi technique: myths and realities. J Adv Nurs 2003;41(4):376-382.

21. Westermeyer J, Janca A. Language, culture and psychopathology: conceptual and methodological issues. Transcultural Psychiatry 1997;34(3):291-311.

22. Van Ommeren M, Sharma B, Thapa S, Makaju R et al. Preparing Instruments for Transcultural Research: Use of the translation monitoring form with Nepali-speaking Bhutanese refugees. Transcultural Psychiatry 1999;36(3):285-301.

23. Wild D, Grovo A, Martin M, Eremenco S et al. Principles of good practice for the translation and cultural adaptation process for patient-reported outcomes (PRO) measures: report of the ISPOR task force for translation and cultural adaptation. Value Health 2005;8(2):94-104.

24. Kleinman A. Anthropology and psychiatry. The role of culture in cross-cultural research on illness. Br J Psychiatry 1987;151:447-454.

25. Martínez-Hernáez A. Cuando las hormigas corretean por el cerebro: retos y realidades de la psiquiatría cultural. Cad Saúde Pública 2006;22(11):2269-2280.

26. De Jong J, Van Ommeren M. Toward a Culture-informed Epidemiology: Combining Qualitative and Quantitative Research in Transcultural Contexts. Transcultural Psychiatry 2002;39(4):422-433.

27. Sperber AD. Translation and validation of study instruments for cross-cultural research. Gastroenterology 2004;126:s124-s128.

28. Herdman M, Fox-Rushby J, Badía X. A model of equivalence in the cultural adaptation of HRQoL instruments: the universalist approach. Qual Life Res 1998;7(4):323-335.

29. Manson SM. Cross-cultural and multi-ethnic assessment of trauma. En: Wilson JP, Keane TM (eds). Assessing psychological trauma and PTSD: A handbook for practitioners. New York: Guilford; 1997.

30. Link BG, Cullen FT, Struening E, Shrout PE et al. A modified labeling theory approach to mental disorders: an empirical assessment. Am Sociol Rev 1989;54(3):400-423.

31. Ritsher JB, Otilingam PG, Grajales M. Internalized stigma of mental illness: psychometric properties of a new measure. Psychiatry Res 2003;121(1):31-49.

32. Cohen J, Struening E. Opinions about mental illness in the personnel of two mental hospitals. J Abnorm Soc Psychol 1962;64(5):349-360.

33. Link BG, Lawrence HY, Phelan J, Collins PY. Measuring mental illness stigma. Schizophr Bull 2004;30(3):511-541.

34. Yllá L, Gonzalez-Pinto A, Ballesteros J, Guillén V. Evolución de las actitudes de la población frente al enfermo mental. Actas Esp Psiquiatr 2007;35(5):323-335.

35. Flores F, Chapa A, Almanza M, Gómez A. Adaptación del programa de intervención "Relaciones saludables" a grupos de México que viven con el virus de la inmunodeficiencia humana. Acta Psiquiát Psicol Am Lat 2011;57(1):29-38.

36. Mora-Ríos J, Medina-Mora ME, Natera G. Metodologías cualitativas para la adaptación de tres instrumentos de estigma hacia la enfermedad mental. $13^{\circ}$ Encuentro Nacional de Investigadores Comisión Coordinadora de Institutos Nacionales de Salud y Hospitales de Alta Especialidad. Oaxaca, México: 17-19 octubre 2008.

37. Boulkedid R, Abdoul H, Loustau M, Sibony $O$ et al. Using and reporting the Delphi method for selecting healthcare quality indicators: A systematic review. PLoS ONE 2011;6(6):e20476.

Artículo sin conflicto de intereses 